\title{
QUANTIZATION OF CLOSED MINI-SUPERSPACE MODELS AS BOUND STATES
}

\author{
J. H. Kung \\ Harvard-Smithsonian Center for Astrophysics \\ 60 Garden Street \\ Cambridge, MA 02138
}




\begin{abstract}
Wheeler-DeWitt equation is applied to $k>0$ Friedmann Robertson Walker metric with various types of matter. It is shown that if the Universe ends in the matter dominated era (e.g., radiation or pressureless gas) with zero cosmological constant, then the resulting Wheeler-DeWitt equation describes a bound state problem. As solutions of a non-degenerate bound state system, the eigen-wave functions are real (Hartle-Hawking) and the usual issue associated with the ambiguity in the boundary conditions for the wave functions is resolved. Furthermore, as a bound state problem, there exists a quantization condition that relates the curvature of the three space with the energy density of the Universe. Incorporating a cosmological constant in the early Universe (inflation) is given as a natural explanation for the large quantum number associated with our Universe, which resulted from the quantization condition.

It is also shown that if there is a cosmological constant $\Lambda>0$ in our Universe that persists for all time, then the resulting Wheeler-DeWitt equation describes a non-bound state system, regardless of the magnitude of the cosmological constant. As a consequence, the wave functions are in general complex (Vilenkin) and the initial conditions for wave functions are a free parameters not determined by the formalism.
\end{abstract}




\section{INTRODUCTION}

It is well known that the Hamiltonian formulation of classical general relativity is a constraint system [1,2]. Mathematically, the existence of constraints can be understood as a consequence of the fact that not all the components of the metric tensor $g_{\mu, \nu}$ are not dynamical coordinates. In the Arnowitt, Deser, Misner (ADM) decomposition of the space-time metric [3], the constraints arise because canonical momenta conjugate to lapse function and shift vectors vanish identically. Quantization of a system via Hamiltonian goes under the name of canonical quantization, in which one has the option of using either Schrodinger or Heisenberg representation of operators. Vanishing of the momentum conjugate to the lapse function, in Schrodinger representation, gives the celebrated Wheeler-DeWitt equation $[4,5]$. Here, operators act on "superspace" that is an infinite-dimensional space of all possible 3-geometries, which are not related by diffeomorphism, and all matter field configurations. In practice, to make problems analytically tractable, the infinite dimensional superspace is reduced to a finite dimensional mini-superspace by working in models with idealized symmetries [6]. In cosmology, the metric of primary interest is that of Friedmann Robertson Walker (FRW) Universe. The gravitational sector of the corresponding mini-superspace models have only one degree of freedom, which is the scale factor of the Universe.

The operator ordering problem, which arises in the formalism, has been addressed in the literature [7-9]. Halliwell [7] and Misner [8] have demonstrated that the operator ordering ambiguity in the Wheeler-DeWitt equation can be completely fixed by demanding invariance under field redefinition of both the three-metric and the lapse function for $D>1$, where $D$ is the number of gravitational coordinates in a mini-superspace model. In semiclassical calculations, operator ordering is usually dictated by convenience. This is because a wrong factor ordering introduces an error that is in higher power of Planck's constant.

A scalar field is usually used in the matter sector of the Wheeler-DeWitt equation [10-13]. This is because one is particularly interested in applying the Wheeler-DeWitt equation to elucidate properties of our early Universe near the Planck epoch, when classical description 
of matter and gravity must necessarily break down.

Because the Wheeler-DeWitt equation is a second order differential equation, a priori the initial conditions (or boundary conditions) are not determined by the formalism and must be specified to completely describe a system. Hartle and Hawking [14,15] have proposed "no boundary" boundary condition, for which the resulting wave functions are real. Hartle-Hawking condition is essentially a prescription for selecting four-manifolds that will be included in the Euclidean path integral over history. On the other hand, Vilenkin $[16,17]$ has proposed a "tunneling" boundary condition, for which the resulting wave functions are complex. Vilenkin's condition is a proposal that the wave function should consist solely of outgoing waves from the initial singularity.

In this paper, we go against the conventional wisdom and first study the mini-superspace model corresponding to closed FRW metric with zero cosmological constant and $\rho=\rho_{\text {rad }}+$ $\rho_{\text {dust }}$. Several comments are in order. First, it is not physically inane to use a classical description of matter in a quantum mechanical equation. The situation is analogous to solving for atomic wave functions while using a classical coulomb interaction. Second, because of the usage of classical description of matter, the resulting wave functions are adequate in describing only the late phase of a universe. There are several motivations for studying this particular model. First, the formalism for quantum cosmology is still in its rudimentary stage, e.g., the role of time, interpretation of wave functions, usage of Euclidean continuation, etc. are not clear [18]. Since the correct interpretation of the theory should give the classical limit, we feel that it is necessary to look into the consequences of the formalism in this classical regime. Second, limiting ourselves to a closed FRW metric is for a technical reason. Of the three possible FRW metrics, i.e., closed, flat, and open, only the closed FRW metric has a finite action. Third, even though this is a classical regime, application of quantum mechanical formalism can lead to interesting results. Indeed, because the resulting Wheeler-DeWitt equation resembles a bound state problem, the quantization condition gives an interesting relation between the energy density of the Universe and the curvature 
of the three space. Fourth, if our Universe is a closed universe that has gone through an inflationary phase, in its early epoch but with zero cosmological constant today, then we may be able to say something about the nature of a wave function immediately following the inflationary phase by invoking the continuity of the wave function and its derivative. Here, we get the conclusion that the appropriate boundary condition for a wave function is that of Hartle and Hawking. The conclusion is based on the fact that eigen-wave functions for a nondegenerate bound state system are necessarily real.

The layout of the paper is as follows. In Sec. II, some notations and basic results are quoted from the literature. In Sec. III, we solve Wheeler-DeWitt equation in a closed form for the closed FRW metric with $\rho=\rho_{\text {rad }}$ to get the complete set of eigen-wave functions. The analysis is carried out for the Laplacian and for an arbitrary operator ordering. In Sec. IV, the analysis is repeated for the more general case of $\rho=\rho_{\text {rad }}+\rho_{\text {dust }}$. In Sec. V, we study the consequences of including a cosmological constant to the Wheeler-DeWitt equation for the $k>0$ FRW metric with $\rho=\rho_{\text {rad }}+\rho_{\text {dust }}$. In Sec VI, we briefly discuss generalization of including more gravitational degree of freedom and its anticipated effects on the nature of wave functions and their corresponding boundary conditions.

\section{PRELIMINARIES}

The starting point is action for the system,

$$
I=I_{M}+I_{G}
$$

$I_{M}$ is an action for matter, and

$$
I=-\frac{1}{16 \pi G} \int d^{4} x \sqrt{-g} R
$$

is the familiar Einstein's action for gravity. We will be primarily interested in the quantum mechanics of FRW metric. 


$$
d s^{2}=-d t^{2}+a^{2}\left(\frac{d r^{2}}{1-k r^{2}}+r^{2} d \Omega_{3}^{2}\right),
$$

where $(a)$ is the scale factor of the Universe, $d \Omega_{3}^{2}$ is the metric on a unit three-sphere, and $k>0, k=0, k<0$ correspond to closed, flat, and open geometry, respectively.

The "time-time" component of Einstein's equation gives

$$
\dot{a}^{2}+k=\frac{8 \pi G}{3} \rho a^{2}
$$

For FRW metric, the Ricci scalar is

$$
R=-6\left(\frac{\ddot{a}}{a}+\left(\frac{\dot{a}}{a}\right)^{2}+\frac{k}{a^{2}}\right)
$$

For the closed FRW metric, the integral over the spatial coordinates in the expression for $I_{G}$ (2.2) is finite and can be explicitly done.

$$
I_{G}=-\frac{3 V_{3}}{8 \pi G k^{3 / 2}} \int d t a\left(\dot{a}^{2}-k\right)
$$

where $V_{3}=2 \pi^{2}$ is the volume of a three dimensional space with constant positive $(k=1)$ curvature. We have also integrated by parts. An astute reader may question the justification for integrating by parts. The reasoning is quite involved $[2,19]$. In turns out that there are two methods of deriving Einstein's equations (e.g. (2.4)) from the principle of least action. In the first method, when the action $(2.2)$ is varied, one needs to require that both $\delta \dot{g}_{\mu \nu}=0$ and $\delta g_{\mu \nu}=0$ at appropriate boundaries in order for all the resulting surface terms to vanish. This is because, in general, the Ricci scalar involves terms with $\ddot{g}_{\mu \nu}$.

In the second method, one starts with an action that differs from (2.2) by a term that is proportional to the trace of the second fundamental form at the boundary. Here, to get Einstein's equation, one only needs to require that $\delta g_{\mu \nu}$ vanish on all appropriate boundaries when the new action is varied. Mathematically, the addition of the new term to the action 
can be viewed as an alternative way of enforcing all the surface terms to vanish in the variation.

In the Hamiltonian formulation of general relativity, it is the latter action that is preferred because of the nature of the variational conditions. For the closed FRW metric, the resulting action for gravity is precisely (2.6). So finally, the justification for integrating by part is that in actuality we are using the latter action.

From (2.6), the canonical momentum conjugate to scale factor is

$$
\pi_{a} \equiv \frac{\delta I_{G}}{\delta \dot{a}}=-\frac{3 V_{3}}{4 \pi G k^{3 / 2}} a \dot{a}=-\frac{3 \pi}{2 G k^{3 / 2}} a \dot{a} .
$$

In terms of $\pi_{a}$, the "time-time" component of Einstein's equation (2.4) is

$$
\pi_{a}^{2}+\left(\frac{3 \pi}{2 G}\right)^{2} \frac{1}{k^{3}}\left[k a^{2}-\frac{8 \pi G}{3} \rho a^{4}\right]=0 .
$$

We will not need the expression for the Wheeler-DeWitt equation in its most general form. To quantize the system, we replace $\pi_{a} \rightarrow-i \frac{\partial}{\partial a}$ in (2.8) to get

$$
\left[-a^{-p} \frac{\partial}{\partial a} a^{p} \frac{\partial}{\partial a}+\left(\frac{3 \pi}{2 G}\right)^{2} \frac{1}{k^{3}}\left(k a^{2}-\frac{8 \pi G}{3} \rho a^{4}\right)\right] \psi(a)=0 .
$$

The parameter $\mathrm{p}$ represents the ambiguity in the ordering of $a$ and $\frac{\partial}{\partial a} \cdot p=1$ is the Laplacian ordering $[9,20,21]$. In the subsequent use of WKB approximation, a convenient $p=2$ will be used.

To describe the matter sector, we substitute

$$
\frac{8 \pi G}{3} \rho \rightarrow \frac{8 \pi G}{3}\left[\rho_{r}\left(\frac{a_{o}}{a}\right)^{4}+\rho_{d}\left(\frac{a_{o}}{a}\right)^{3}\right]+\frac{\Lambda}{3},
$$

where $\rho_{r}, \rho_{d}$, and $\Lambda$ are radiation energy density, matter (dust) energy density, and cosmological constant, respectively.

It is convenient to define several dimensionless parameters.

$$
\alpha^{2} \equiv \frac{3 \pi}{2 G k}
$$




$$
\begin{gathered}
\gamma \equiv \frac{8 \pi G}{6 k} \rho_{d} a_{o}^{3} \\
\beta^{2} \equiv\left(\frac{3 \pi}{2 G k}\right)^{2}\left(\frac{8 \pi G}{3 k} \rho_{r} a_{o}^{4}+\left(\frac{8 \pi G}{6 k} \rho_{d} a_{o}^{3}\right)^{2}\right) \\
\mu \equiv\left(\frac{3 \pi}{2 G}\right)^{2} \frac{\Lambda}{3 k^{3}}
\end{gathered}
$$

In terms of these variables the Wheeler-DeWitt equation is

$$
\left[-a^{-p} \frac{\partial}{\partial a} a^{p} \frac{\partial}{\partial a}+\alpha^{4}(a-\gamma)^{2}-\mu a^{4}-\beta^{2}\right] \psi(a)=0 .
$$

We further define rescaled variables.

$$
\begin{gathered}
\tilde{a} \equiv \alpha a \\
\tilde{\beta} \equiv \beta / \alpha \\
\tilde{\gamma} \equiv \alpha \gamma \\
\tilde{\mu} \equiv \mu / \alpha^{6}
\end{gathered}
$$

The Wheeler-DeWitt equation now becomes

$$
\begin{gathered}
{\left[-\tilde{a}^{-p} \frac{\partial}{\partial \tilde{a}} a^{p} \frac{\partial}{\partial \tilde{a}}+2 U(\tilde{a})\right] \psi(\tilde{a})=0 .} \\
2 U(\tilde{a}) \equiv(\tilde{a}-\tilde{\gamma})^{2}-\tilde{\mu} \tilde{a}^{4}-\tilde{\beta}^{2} .
\end{gathered}
$$

III. MODEL WITH $k>0, \rho=\rho_{\text {rad }}$ 
In this section, we would like to study the Wheeler-DeWitt equation for a closed FRW metric with just radiation as matter. There are several reasons for studying this simplified case. First, it is solvable in a closed form. Second, while solving this model, we will encounter general properties of quantizing a bound state system, i.e., existence of a quantization condition and no need for an arbitrary boundary condition for the wave function.

The dimensionless parameters (2.16-2.19) are now $\tilde{\gamma}=\tilde{\mu}=0, \tilde{\beta} \neq 0$, and the WheelerDeWitt equation $(2.20,2.21)$ is

$$
\left[\frac{d^{2}}{d \tilde{a}^{2}}+\frac{p}{\tilde{a}} \frac{d}{d \tilde{a}}-2 U(\tilde{a})\right] \psi(\tilde{a})=0,
$$

with (Fig. 1)

$$
2 U(\tilde{a}) \equiv \tilde{a}^{2}-\tilde{\beta}^{2}
$$

The dominant behavior of $\psi$ for $\tilde{a} \rightarrow \infty$ is $e^{-\tilde{a}^{2} / 2}$. Factoring out the exponential behavior, $\psi(\tilde{a}) \equiv g(\tilde{a}) e^{-\tilde{a}^{2} / 2}, g(\tilde{a})$ satisfies

$$
\frac{d^{2} g}{d \tilde{a}^{2}}+\left(\frac{p}{\tilde{a}}-2 \tilde{a}\right) \frac{d g}{d \tilde{a}}+\left(\tilde{\beta}^{2}-1-p\right) g=0
$$

Power series method reveals that for $p \neq 0$ the function $g(\tilde{a})$ is an even function. Therefore, in terms of $x \equiv \tilde{a}^{2}, g(x)$ satisfies

$$
x \frac{d^{2} g}{d x^{2}}+\left(\frac{1+p}{2}-x\right) \frac{d g}{d x}+\left(\frac{\tilde{\beta}^{2}-1-p}{4}\right) g=0 .
$$

We first seek solution for the Laplacian factor ordering (e.g. $p=1$ ) $[9,20,21]$. For the wave function $\psi$ to remain finite for $x \rightarrow \infty$, the parameter $\tilde{\beta}$ must satisfy a quantization condition

$$
\tilde{\beta}^{2}=4 n+2 \quad n=0,1,2 \ldots
$$

The "eigenfunctions" are the familiar Laguerre polynomials [22] 


$$
g(x)=L_{n=\frac{\tilde{\beta}^{2}-2}{4}}^{0}(x) .
$$

And finally, the wave functions are

$$
\psi_{n}(\tilde{a})=e^{-\tilde{a}^{2} / 2} L_{n=\frac{\tilde{\beta}^{2}-2}{4}}^{0}\left(\tilde{a}^{2}\right) .
$$

In terms of radiation energy density, the quantization condition is

$$
\beta^{2} \equiv\left(\frac{3 \pi}{2 G k}\right)^{2} \frac{8 \pi G}{3 k} \rho_{r} a_{o}^{4}=4 n+2 .
$$

The eigenfunctions $\psi_{n}$, corresponding to first few $n$ states are plotted in Fig. 2 .

Before leaving this model, we would like to investigate the consequences of quantizing the same system for another operator ordering $(p=2)$. For $\rho=\rho_{\text {rad }}$, the $p=2$ ordering is also exactly solvable. The comparison of the two sets of solutions will lend credence to the conventional wisdom that operator ordering may be dictated by convenience.

For $p=2$, the wave equation $(3.1,3.2)$ can be rewritten in a form that resembles a one-dimensional wave equation with a potential. $g(\tilde{a})$ defined as $\psi(\tilde{a})=g(\tilde{a}) / \tilde{a}$ satisfies

$$
\left[\frac{d^{2}}{d \tilde{a}^{2}}-\tilde{a}^{2}+\tilde{\beta}^{2}\right] g(\tilde{a})=0,
$$

which is the familiar Schrodinger equation for a harmonic oscillator. The only difference is that among the complete set of Hermite polynomials, which makes up the solution to harmonic oscillator problem, only the odd Hermite polynomials are acceptable in this case. This is necessary in order for the wave function $\psi(\tilde{a}) \equiv \frac{g(\tilde{a})}{\tilde{a}}$ to remain finite at $\tilde{a}=0$. The familiar quantization condition for harmonic oscillator gives

$$
\tilde{\beta}^{2}=2(\text { odd })+1=4 n+3, \quad n=0,1,2 \ldots
$$

Comparison with the result for the Laplacian ordering (3.5) shows that the eigenvalue spectrum is essentially unchanged. It can also be shown that for an arbitrary $p \geq 1$ operator ordering, the quantization condition is 


$$
\tilde{\beta}^{2}=4 n+1+p, \quad n=0,1,2 \ldots
$$

\section{MODEL WITH $k>0, \rho=\rho_{\text {rad }}+\rho_{\text {dust }}$}

In this section, we would like to study the Wheeler-DeWitt equation for a more realistic case of FRW metric with a combination of radiation and pressureless dust.

The dimensionless parameters (2.16-2.19) are now $\tilde{\gamma} \neq 0, \tilde{\beta} \neq 0$, and $\tilde{\mu}=0$. To keep the model as realistic as possible, we will assume that $\rho_{\text {rad }} / \rho_{\text {dust }} \approx O\left(10^{-4}\right)$ at $a_{o} \equiv 1$, which implies that $\tilde{\beta} \approx \tilde{\gamma}$.

The Wheeler-DeWitt equation $(2.20,2.21)$ is now

$$
\left[\frac{d^{2}}{d \tilde{a}^{2}}+\frac{p}{\tilde{a}} \frac{d}{d \tilde{a}}-2 U(\tilde{a})\right] \psi(\tilde{a})=0
$$

with

$$
2 U(\tilde{a}) \equiv(\tilde{a}-\tilde{\gamma})^{2}-\tilde{\beta}^{2}
$$

A schematic graph of the "potential" $U(\tilde{a})$ is given in Fig. 3. The solution to this differential equation doesn't seem to be analytically tractable for the Laplacian operator ordering $(p=1)$. We will therefore resort to solving the equation for another ordering (i.e., $p=2$ ) where the problem is analytically tractable.

Again for $p=2$, the wave equation $(4.1,4.2)$ can be rewritten in a form that resembles a one-dimensional wave equation with a potential. $g(\tilde{a})$ defined as $\psi(\tilde{a})=g(\tilde{a}) / \tilde{a}$ satisfies

$$
\left[\frac{d^{2}}{d \tilde{a}^{2}}-(\tilde{a}-\tilde{\gamma})^{2}+\tilde{\beta}^{2}\right] g(\tilde{a})=0,
$$

which resembles a simple harmonic oscillator with a shift $\Delta x=-\tilde{\gamma}$ in the potential. The key difference is that here the range of coordinate is $\tilde{a} \in[0, \infty]$ whereas for the harmonic oscillator the range is $[-\infty, \infty]$. 
A solution to (4.3) which vanishes for very large positive values of $\tilde{a}$ is

$$
g(\tilde{a})=D_{\tilde{\beta}^{2}-\frac{1}{2}}(\tilde{a}-\tilde{\gamma})
$$

where $D_{\nu}(z)$ is the parabolic cylinder function [23].

The quantization condition is obtained by requiring that

$$
g(\tilde{a}=0)=D_{\tilde{\beta}^{2}-\frac{1}{2}}(-\tilde{\gamma})=0
$$

This is again necessary in order for the wave function $\psi(\tilde{a}) \equiv g(\tilde{a}) / \tilde{a}$ to remain finite at $\tilde{a}=0$. Disappointingly, it is difficult to calculate the parameters $\tilde{\beta}$, and $\tilde{\gamma}$ for which (4.5) is satisfied. We are left to resort to WKB approximation to find the approximate "eigenvalues". The appropriate WKB phase integral relevant for (4.3) is

$$
J \equiv \int_{0}^{\tilde{\beta}+\tilde{\gamma}}(-2 U(\tilde{a}))^{1 / 2} d \tilde{a}=\pi(n+3 / 4) \quad n=0,1,2 . .
$$

The usual $1 / 2$ is replaced by $3 / 4$ because $\tilde{a}=0$ is not a usual classical turning point but an end of the coordinate, and also because we must demand that $g(\tilde{a}=0)=0$ [24]. For the limiting case of $\tilde{\beta}=\tilde{\gamma}$, which is the statement that $\rho_{\text {rad }}=0$ and $\rho=\rho_{\text {dust }}$, the phase integral can be readily evaluated to give $J=\frac{\pi}{2} \tilde{\gamma}^{2}$.

In terms of $\rho=\rho_{\text {dust }}$, the quantization condition is

$$
\left(\frac{3 \pi}{2 G k}\right)\left(\frac{8 \pi G}{6 k} \rho_{d} a_{o}^{3}\right)^{2}=2(n+3 / 4) .
$$

For our Universe, $\tilde{\beta} \neq \tilde{\gamma}$ but

$$
\frac{\tilde{\beta}^{2}-\tilde{\gamma}^{2}}{\tilde{\gamma}^{2}} \approx \frac{\rho_{r}}{\rho_{d}} \frac{2 k}{H_{0}^{2}+k} \sim O\left(10^{-4}\right) .
$$

In the Appendix , the phase integral for $\tilde{\beta} \neq \tilde{\gamma}$ case is evaluated to give $J \approx \frac{\pi}{2} \tilde{\beta}^{2}$. In terms of $\rho_{\text {rad }}$ and $\rho_{\text {dust }}$, the quantization condition is 


$$
\left(\frac{3 \pi}{2 G k}\right)\left(\frac{8 \pi G}{3 k} \rho_{r} a_{o}^{4}+\left(\frac{8 \pi G}{6 k} \rho_{d} a_{o}^{3}\right)^{2}\right)=2(n+3 / 4)
$$

If we make a rough estimate that $k \sim H_{o}^{2}$, then the quantum number of our Universe is

$$
N \sim(G k)^{-1} \sim\left(\frac{\text { three radius }}{\text { Planck length }}\right)^{2} \sim\left(\frac{H_{o}^{-1}}{\text { Planck length }}\right)^{2} \sim 10^{122} .
$$

A comment is in order. It is obvious that the entire analysis was facilitated by the use of $p=2$ operator ordering. It is also easy to convince oneself that even if we could solve for the $p=1$ operator ordering the two quantization results should not differ by much. The argument goes as follows. Because the operator ordering parameter $(p)$ appears with $\tilde{a}^{-1}$ in the Wheeler-DeWitt equation (4.1), a wrong value for $p$ should affect the small range of $\tilde{a}$ the most. But for a universe with a combination of radiation and dust, small value for the scale factor corresponds to the radiation dominated era. And since the two exact quantization conditions for $p=1,2$ orderings with $\rho=\rho_{\text {rad }}$ are essentially the same $(3.5,3.10)$, we can also safely conclude that the quantization conditions for $p=1,2$ orderings should not differ by much even for the present case.

Interestingly, the unusual largeness of the quantum number of our Universe can be easily explained within the framework of inflationary universe models. This will be addressed in the next section.

\section{MODEL WITH $k>0, \rho=\rho_{\text {rad }}+\rho_{\text {dust }}$, and $\Lambda \neq 0$}

In this section, we would like to study the effects of including a cosmological constant to a FRW universe which in its late stage of evolution becomes radiation and then matter dominated. A straightforward method, at least formally, would be to include a spatially homogeneous scalar field $\phi$ with some self coupling $V(\phi)$, whose non-zero expectation value would play the role of a cosmological constant [25]. Moreover, as a reheating mechanism, there should also be some coupling between the scalar field and the radiation field, so that after the $e^{60}$ expansion the scalar field could transfer its vacuum energy to the radiation field. 
Needless to say this is a very tall order. Here we make a loose-shoe approximation by putting in by hand a cosmological constant $\Lambda_{\mathrm{GUT}}$. And to simulate the reheating mechanism, we set $\Lambda_{\mathrm{GUT}}=8 \pi G \rho_{\mathrm{rad}}$ at some high redshift (e.g., $z=z_{\mathrm{GUT}}$ ) and then set $\Lambda_{\mathrm{GUT}}=0$ for $z \leq z_{\mathrm{GUT}}$. The Wheeler-DeWitt equation is now (2.16-2.21)

$$
\left[\frac{d^{2}}{d \tilde{a}^{2}}+\frac{p}{\tilde{a}} \frac{d}{d \tilde{a}}-2 U(\tilde{a})\right] \psi(\tilde{a})=0,
$$

with

$$
2 U(\tilde{a})= \begin{cases}\tilde{a}^{2}-\tilde{\mu} \tilde{a}^{4} & \mathrm{z} \geq \mathrm{z}_{\mathrm{GUT}} \\ (\tilde{a}-\tilde{\gamma})^{2}-\tilde{\beta}^{2} & \mathrm{z} \leq \mathrm{z}_{\mathrm{GUT}}\end{cases}
$$

A schematic plot of $U(\tilde{a})$ is given is Fig. 4. From the figure, we can readily conclude that it is again a bound state problem. Moreover, we can immediately infer an essential difference between including and not including the reheating phase. If there were no reheating but a constant $\Lambda \neq 0$ for all time, then the potential $U(\tilde{a})$ would describe a non-bound state problem Fig. 5. Moreover, $\Lambda \rightarrow 0$ has a unique role in the parameter space. Intuitively, if $\Lambda_{\mathrm{GUT}}$ settles to some small but a finite value $\Lambda_{\text {final }}$, then the metric can re-enter into a de-Sitter phase, albeit the two de-Sitter phases may be separated by a classically forbidden region. Mathematically, this is because $\Lambda$ appears in $U(\tilde{a})$ with the $\tilde{a}^{4}$ factor, which eventually comes to dominate $U(\tilde{a})$. In the Wheeler-DeWitt formalism, this would correspond to a tunneling model, which is analogous to a quantum mechanical description of Alpha decay of a heavy nucleus. A schematic plot of classically allowed regions in $U(\tilde{a})$ for this case is given in Fig. 6.

If we assume that we live in this classical allowed region with a small $\Lambda_{\text {final }} \sim O(1) H_{o}^{2}$, then it is very interesting to estimate the tunneling probability. The Euclidean action for tunneling region is [26]

$$
S_{E} \approx-\left(G \Lambda_{\text {final }}\right)^{-1} \approx-G^{-1} H_{o}^{-2} \approx-122
$$


And the tunneling probability is [27-30]

$$
P_{T} \propto \exp \left(-\left|S_{E}\right|\right) \sim \exp (-122) .
$$

Now let us return to the case where $\Lambda_{\mathrm{GUT}} \neq 0$ only at large redshifts (e.g. $z \geq z_{\mathrm{GUT}}$ ) and $\Lambda_{\text {final }}=0$. We are interested in its effect on the quantization condition (4.9). It is easy to trace the large $n \sim 10^{122}$ back to the factor $G k$. In light of this fact, having a cosmological constant in our early universe can be interpreted as the mechanism which "generated" this large quantum number. This is because one of the triumph of an inflationary universe model is its ability to naturally explain the flatness of the observed Universe.

Finally, let us investigate the nature of $\psi$ at early Universe. For $z \ll z_{\mathrm{GUT}}$, the wave function should be that of $\rho=\rho_{\text {rad }}+\rho_{\text {dust }}$ model (4.4). For $z \ll z_{\text {GUT }}$ Wheeler-DeWitt equation for the Laplacian ordering is $(5.1,5.2)$

$$
\left[\frac{d^{2}}{d \tilde{a}^{2}}+\frac{1}{\tilde{a}} \frac{d}{d \tilde{a}}+\tilde{a}^{2}-\tilde{\mu} \tilde{a}^{4}\right] \psi(\tilde{a})=0 .
$$

Moreover, near $\tilde{a} \approx 0$ the cosmological constant term is negligible compare to the curvature term. For this region, the differential equation has a single independent solution

$$
\psi(\tilde{a})=I_{0}\left(\tilde{a}^{2} / 2\right) .
$$

$I_{\nu}(x)$ is a modified Bessel functions [22]. Its power series expansion is

$$
I_{\nu}(x)=\sum_{s=0}^{\infty} \frac{1}{s !(s+\nu) !}\left(\frac{x}{2}\right)^{2 s+\nu} .
$$

Interestingly, $\psi(\tilde{a}=0) \neq 0$ because $I_{0}(0)=1$.

There are also inflationary universe models where $\rho_{\text {rad }} \neq 0$ before the inflationary phase $[31,32]$. In such a case, the Wheeler-DeWitt equation for $z \geq z_{\mathrm{GUT}}$ is (2.16-2.21)

$$
\left[\frac{d^{2}}{d \tilde{a}^{2}}+\frac{1}{\tilde{a}} \frac{d}{d \tilde{a}}+\tilde{a}^{2}-\tilde{\mu} \tilde{a}^{4}-\tilde{\beta}^{2}\right] \psi(\tilde{a})=0 .
$$


Again near $\tilde{a} \approx 0$ the cosmological constant term is negligible. The resulting differential equation has a single independent solution (3.7)

$$
\psi_{n}(\tilde{a})=e^{-\tilde{a}^{2} / 2} L_{n=\frac{\tilde{\beta}^{2}-2}{4}}^{0}\left(\tilde{a}^{2}\right) .
$$

And because $L_{n}(0)=1$ the wave function $\psi_{n}(\tilde{a}=0) \neq 0$.

\section{DISCUSSION}

In this section we would like to briefly discuss an interesting generalization. Consider a $(k>0)$ mini-superspace model, which ends in $\rho=\rho_{\text {rad }}+\rho_{\text {dust }}$, but now the corresponding Wheeler-DeWitt Hamiltonian is invariant under some symmetry transformation by having more than one gravitational degree of freedom, e.g., homogeneous but anisotropic Kasner metric [33-35]. In general, symmetry is a sign of degenerate in eigenvalues. In such a case we would have an interesting consequence. First, the problem is still a bound state, so there would be no need for an arbitrary boundary condition when solving for the wave functions. But from general quantum mechanical considerations, eigen-wave functions for a bound state is guaranteed to be real only if there isn't any degeneracy. So we have a combination of Hartle-Hawking's no boundary condition with Vilenkin's complex wave functions with it non-zero flux, $J=\frac{1}{2}\left(\phi \nabla \psi^{*}-\psi^{*} \nabla \psi\right)$. The situation would be analogous to $n^{2}$ degeneracy for each $E_{n}$ level in a hydrogen atom.

\section{CONCLUSION}

In this paper, we have studied the solutions to Wheeler-DeWitt equation for $k>0$ Friedmann Robertson Walker metric with various types of matter. First, we have shown that if the Universe ends in the matter dominated era, then the resulting Wheeler-DeWitt equation describes a bound state problem. As solutions of a non-degenerate bound state system, the eigen-wave functions are real (Hartle-Hawking) and the usual issue associated with the ambiguity in the boundary conditions for the wave functions is resolved. Furthermore, as a bound 
state problem, there exists a quantization condition that relates the curvature of the three space with the energy density of the Universe. Incorporating a cosmological constant in the early Universe (inflation) was given as a natural explanation for the large quantum number our Universe, which resulted from the quantization condition. Second, we have shown that if there is a cosmological constant $\Lambda>0$ in our Universe that persists for all time, then the resulting Wheeler-DeWitt equation describes a non-bound state system, regardless of the magnitude of the cosmological constant. As a consequence, the wave functions are in general complex (Vilenkin) and the initial conditions for wave functions are free parameters not determined by the formalism.

\section{APPENDIX}

We would like to show that

$$
J \equiv \int_{0}^{\tilde{\beta}+\tilde{\gamma}} p d x \approx \frac{\pi}{2} \tilde{\beta}^{2}
$$

with

$$
p=\left(\tilde{\beta}^{2}-(\tilde{\gamma}-\tilde{a})^{2}\right)^{1 / 2}
$$

For our Universe

$$
\frac{\tilde{\beta}^{2}-\tilde{\gamma}^{2}}{\tilde{\gamma}^{2}} \approx \frac{2 k}{H_{0}^{2}+k} \frac{\rho_{\mathrm{rad}}}{\rho_{\mathrm{dust}}} \sim O\left(10^{-4}\right) .
$$

Therefore, defining $x=\tilde{a} / \tilde{\gamma}$, we have

$$
\begin{aligned}
J & =\int_{0}^{\tilde{\beta}+\tilde{\gamma}}\left(\tilde{\beta}^{2}-(\tilde{\gamma}-\tilde{a})^{2}\right)^{1 / 2} d x \\
& =\int_{\tilde{\gamma}}^{\tilde{\beta}} \tilde{\gamma}^{2}\left(\left(\frac{\tilde{\beta}}{\tilde{\gamma}}\right)^{2}-\left(\frac{\tilde{a}}{\tilde{\gamma}}\right)^{2}\right)^{1 / 2} d\left(\frac{\tilde{a}}{\tilde{\gamma}}\right) \\
& \approx \tilde{\gamma}^{2} \int_{-1}^{1}\left(\left(\frac{\tilde{\beta}}{\tilde{\gamma}}\right)^{2}-x^{2}\right)^{1 / 2} d x
\end{aligned}
$$




$$
\begin{aligned}
& \approx \tilde{\gamma}^{2} \int_{-1}^{1}\left[\left(1-x^{2}\right)^{1 / 2}+\frac{1}{2} \frac{\left(\frac{\tilde{\beta}}{\tilde{\gamma}}\right)^{2}-1}{\left(1-x^{2}\right)^{1 / 2}}\right] d x \\
& \approx \tilde{\gamma}^{2}\left[\pi / 2+\pi / 2\left(\left(\frac{\tilde{\beta}}{\tilde{\gamma}}\right)^{2}-1\right)\right] \\
& \approx \frac{1}{2} \pi \tilde{\beta}^{2} .
\end{aligned}
$$

\section{ACKNOWLEDGMENTS}

I would like to thank George Field, Christina Doyle, Eric Blackman, Min Yan for making my extended stay at the Center for Astrophysics so pleasant. 


\section{FIGURE CAPTIONS}

1. Graph of $U(\tilde{a})$ for $\rho=\rho_{\text {rad }}$ and $k>0$.

2. Graphs of $\psi_{n}(\tilde{a})$ for first few quantum states. Figures $2(\mathrm{a})-2(\mathrm{~d})$ correspond to $n=$ $[0,1,2,20]$, respectively. The normalization is arbitrary.

3. Graph of $U(\tilde{a})$ for $\rho=\rho_{\text {rad }}+\rho_{\text {dust }}$ and $k>0$.

4. Graph of $U(\tilde{a})$ for $\rho=\rho_{\text {rad }}+\rho_{\text {dust }}, k>0$, and $\Lambda_{\mathrm{GUT}} \neq 0$ for $z \geq z_{\mathrm{GUT}}$.

5. Graph of $U(\tilde{a})$ for $k>0$ and $\Lambda \neq 0$ for all time.

6. Graph of $U(\tilde{a})$ for $\rho=\rho_{\text {rad }}+\rho_{\text {dust }}, k>0, \Lambda_{\mathrm{GUT}}$, and $\Lambda_{\text {final }}$. $\Lambda_{\mathrm{GUT}}$ is responsible for inflation and $\Lambda_{\text {final }}$ is some final value the cosmological constant has settled into in a universe. 


\section{REFERENCES}

1. P. A. M Dirac, Lectures on Quantum Mechanics (Belfer Graduate School of Science Monographs Number Two, Yeshiva University, New York, 1964).

2. R. M. Wald, General Relativity (Univ. of Chicago Press, Chicago, 1984).

3. R. Arnowitt, S. Deser, and C. W. Miser, in Gravitation: An Introduction to Current Research (Wiley, New York, 1963).

4. B. S. DeWitt, Phys. Rev. 160, 1113 (1967).

5. J. A. Wheeler, in Battelle Rencontres, edited by C. DeWitt and J. A. Wheeler (Benjamin, New York, 1968).

6. C. W. Misner, in Magic Without Magic: John Archibald Wheeler, edited by J.R. Klauder (Freeman, San Francisco, 1972).

7. J. J. Halliwell, Phys. Rev. D 38, 2468 (1988).

8. C. W. Misner, in Relativity, edited by M Carmeli, S. I. Fickler, and L. Witten (Plenum, New York, 1970).

9. S. W. Hawking and D. N. Page, Nucl. Phys. B 264, 185 (1986).

10. W. F. Blyth and C. J. Isham, Phys. Rev. D 11, 768 (1975).

11. T. Christodoulakis and J. Zanelli, Phys. Lett. 102 A, 227 (1984).

12. G. Esposito and G. Platania, Class. Quantum Grav. 5, 937 (1988).

13. G. W. Gibbons and L. P. Grishchuk, Nucl. Phys. B313, 736 (1988).

14. J. B. Hartle and S. W. Hawking, Phys. Rev. D 28, 2960 (1983).

15. S. W. Hawking, Nucl. Phys. B 239, 257 (1984). 
16. A. Vilenkin, Phys. Rev. D 33, 3560 (1986).

17. A. Vilenkin, Phys. Rev. D 37, 888 (1986).

18. E. Alvarez, Rev. Mod. Phys. 61, 561 (1989).

19. C. W. Misner, K. S. Thorne, and J. A. Wheeler, Gravitation (Freeman, San Francisco, 1973).

20. G. A. Ringwood, J. Phys. A: Math. Gen., 9, 1253 (1976).

21. K. S. Cheng, J. Math. Phys., 13, 1723 (1972).

22. G. Arfken, Math. Methods for Physicists (Academic, New York, 1970).

23. I. S. Gradshteyn and I. M Ryzhik, Table of Integral, Series, and Products (Academic, New York, 1965).

24. E. Merzbacher, Quantum Mechanics (Wiley, New York, 1970).

25. A. Guth, Phys. Rev. D 23, 347 (1981).

26. E. W. Kolb and M. S. Turner, The Early Universe (Addison-Wesley, New York, 1990).

27. A. D. Linde, JETP 60, 211 (1984).

28. A. Vilenkin, Phys. Rev. D 30, 549 (1984).

29. V. A. Rubakov, Phys. Lett. 148 B, 280 (1984).

30. Ya. B. Zel'dovich and A. A. Starobinskii, Sov. Astron. Lett. 10, 135 (1984).

31. A. Albrecht and R. Brandenberger, Phys. Rev. D 31, 1225 (1985).

32. J. H. kung and R. Brandenberger, Phys. Rev. D 40, 2532 (1989).

33. E. Kasner, Am. J. Math. 43, 217 (1921). 
34. E. Schucking and O. Heckmann, "World models," in Onzieme Conseil de Physique Solvay, Editions Stoops, Brussels (1958).

35. C. W. Misner, Phys. Rev. Lett. 22, 1071 (1969). 